\title{
Alexia without agraphia
}

\author{
D.J.Quint ${ }^{1}$ and J.L. Gilmore ${ }^{2}$ \\ ${ }^{1}$ Division of Neuroradiology, Department of Radiology, University of Michigan Hospitals, Ann Arbor, Michigan, USA \\ ${ }^{2}$ Department of Neurology, University of Rochester, Rochester, New York, USA
}

Received: September 16, 1991

Summary. Two new cases of alexia without agraphia are presented. Pertinent clinical findings, anatomy, pathophysiology and differential diagnoses are reviewed. The importance of carefully examining the inferior portion of the left side of the splenium of the corpus callosum on CT and/or MR scans in patients who present with this clinical syndrome is stressed.

Key words: Alexia - Agraphia - Disconnection syndrome - Magnetic resonance imaging - Computed tomography

Alexia without agraphia or pure word blindness is considered one of the classic disconnection syndromes. Patients retain the ability to write, but are unable to read (even words that they have just written) and often have right homonymous hemianopia. This disorder results from the loss of visual input to the language area [usually the dominant (left) angular gyrus] without involvement of the language area or output from the language area to the motor cortex. The ability to process visual input into language (i.e., reading) is lost in these patients. The pathways involved in this syndrome are believed to be localized to the inferior portion of the splenium of the corpus callosum on the side of the dominant (usually left) cerebral hemisphere. Therefore, head CT and MR examinations in patients who present with this symptom complex should be scrutinized for abnormalities in this region.

This report of two additional cases of alexia without agraphia is presented to show what we believe to be the first published example of this syndrome demonstrated on MRI and to stress the importance of understanding the underlying anatomy thought to be involved so that the appropriate regions are studied.

\section{Case reports}

\section{Case 1}

A 77-year-old right-handed woman with a long history of atherosclerotic peripheral vascular disease and scleroderma presented to the psychiatry service with decreased mentation and right visual complaints. She had suffered from extensive atherosclerotic peripheral vascular disease involving both the systemic and cerebral vasculature, necessitating carotid endarterectomy. The patient denied any "strokes", but did suffer from complications of scleroderma including gastritis, esophagitis and Raynaud's phenomenon and had also had a cervical sympathectomy. The patient did report a vague episode of "loss of consciousness" 4-5 months before admission.

General physical examination showed some edema of the legs. Neurologic examination revealed the patient to be oriented to person and place, but not to time. She could identify letters, but could not read. She could write her name and spell simple wordsforwards and backwards. Cranial nerve (II-XII) examination demonstrated a right homonymous hemianopia. Cerebellar function tests demonstrated decreased rapid alternating movements on the left.

Laboratory studies, with the exception of mild elevation of BUN and creatinine, were normal. The EEG demonstrated slowing over the left posterior temporal and occipital regions consistent with a structural disturbance. Mild right temporal slowing was also demonstrated. Head CT (Fig. 1) demonstrated a left occipital infarct.

Formal neuropsychometric testing confirmed alexia without agraphia.

\section{Case 2}

A 79-year-old right-handed man with a history of hypertension was otherwise well until 2 months prior to admission when he experienced an episode of "jamais vu": he could recognize his surroundings, but they seemed unfamiliar to him. This episode lasted several days and recurred 1 month prior to admission. The patient experienced an additional episode of "feeling odd" which prompted his admission to hospital 2 days later. While the patient did report a 2-month history of intermittent loss of right vision, diplopia, vertigo, nausea and problems with speech, he denied loss of consciousness, seizures, episodes of weakness or symptoms consistent with stroke. Apart from the history of elevated blood pressure, his past medical history was unremarkable. 

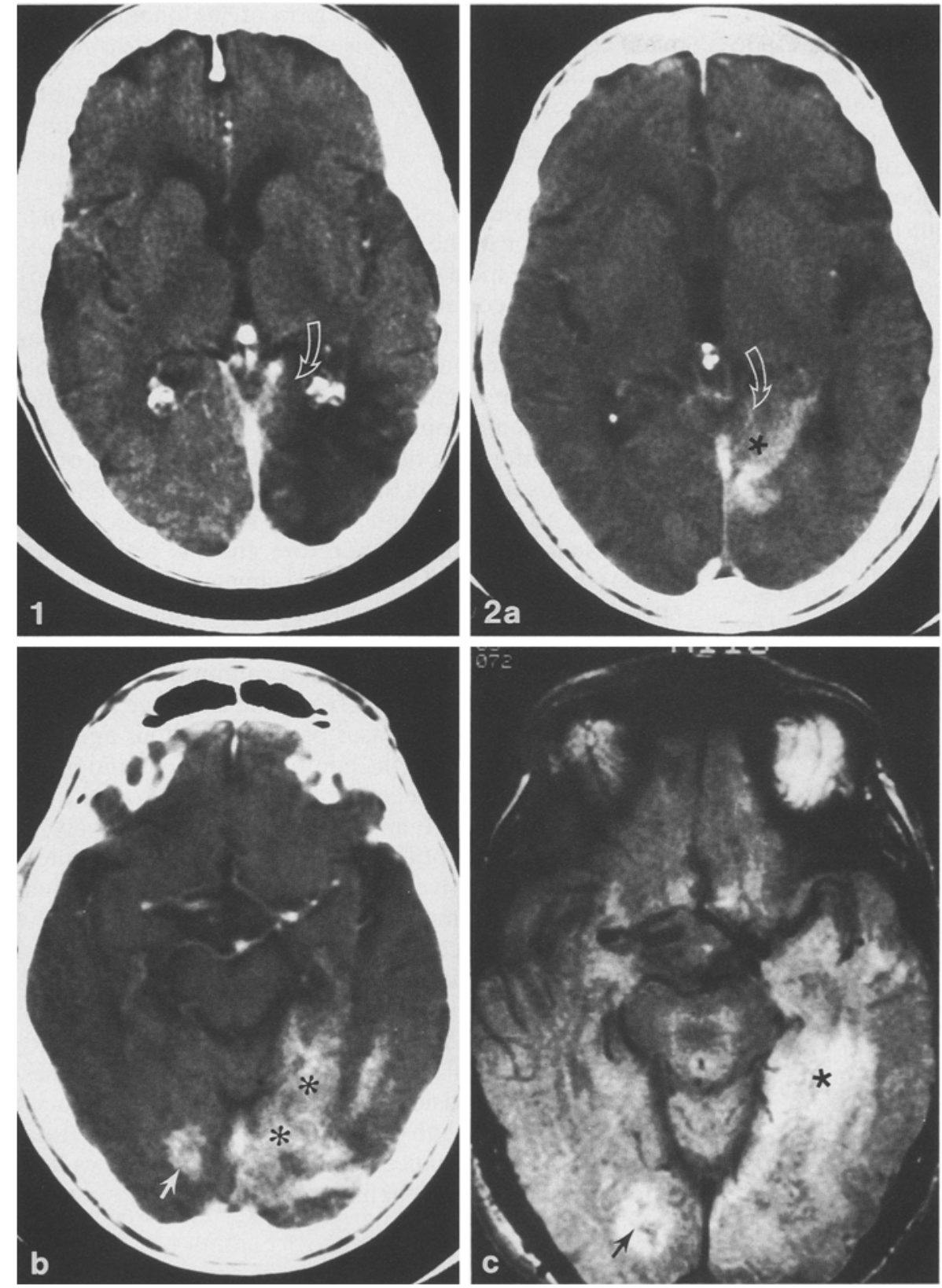

Fig.1. Case 1.77-year-old right-handed woman with alexia without agraphia and right homonymous hemianopia. CT demonstrates decreased attenuation with mild mass effect in the left posterior cerebral artery territory consistent with subacute infarction. Curved arrow indicates inferior portion of left side of splenium of corpus callosum

Fig.2a-c. Case 2.79-year-old righthanded man with alexia without agraphia and right homonymous hemianopia. Contrast-enhanced CT demonstrates homogeneous contrast enhancement of a subacute left posterior cerebral artery territory infarct involving a the left side of the inferior portion of the splenium of the corpus callosum (curved arrow) and b the left parieto-occipital and left posteromedial temporal regions (asterisks). A smaller right occipital infarct is also seen (straight arrow). c Proton density weighted MRI (1.5 T, 2000/30) also shows the right occipital and left parieto-occipital and medial temporal lesions
The patient was normotensive. General physical examination was normal. Neurologic examination revealed the patient to be oriented to person, place and time. Speech was normal without dysarthria. The patient was unable to read, although neuropsychometric testing revealed that he could identify individual letters. He was unable to name objects and to perform simple (seventh grade education) arithmetic. He could recognize colors and write and did not demonstrate left/right confusion. He was able to follow simple commands. Cranial nerve (II-XII) examination demonstrated a right homonymous hemianopia, decreased pin-prick and touch sensation in the distribution of all three divisions of the left trigeminal nerve and a right central facial weakness. Sensory examination demonstrated decreased vibration sense in the lower limbs bilaterally (left more than right) and decreased pin-prick and touch sensation on the right side of the body. Finger- to-nose movements were decreased on the left and there was mild gait ataxia.

Laboratory studies were normal. Head CT (Figs. 2a, b) demonstrated an area of pathologic contrast enhancement in the deep left parieto-occipital region most consistent with a subacute left posterior cerebral artery distribution infarct. MRI (Fig. 2c) confirmed this.

Formal neuropsychometric testing interpretation confirmed alexia without agraphia. Higher cortical function and memory testing suggested additional bilateral cerebral hemisphere dysfunction.

\section{Discussion}

Disconnection syndromes are the result of interruption of inter- or intrahemispheric white matter tracts [1]. Alexia without agraphia is a rare but "classic" disconnection syn- 
drome [2]. Déjerine [3] was the first to perform a postmortem examination on a patient with this syndrome. $\mathrm{He}$ found an infarct of the left posterior cerebral artery territory involving the splenium of the corpus callosum and the medial portion of the left occipital lobe. He and others $[4$, 5] elaborated on the work of Wernicke [6], concluding that loss of visual input from both occipital lobes to the dominant angular gyrus (language area) results in the inability to read without affecting the language area itself or the output from the language region to the motor region (the ability to write and speak).

Over the past 100 years, alexia (the inability to comprehend written material) has been classified in several different ways, which essentially divide it into two major groups: alexia associated with or unassociated with agraphia [7-9]. Recently, the alexias have been further categorized by a neurolinguistic approach specifying the type of reading disability [9]. One such subclassification scheme, that of Marshall and Newcombe [10], has gained widespread acceptance. It divides the alexias into three groups including "visual dyslexia" (misreading a word by transposing letter positions, e.g., "exit" read as "next"), "deep dyslexia" (replacing a word with a synonym, e.g., "car" read as "auto") and "surface dyslexia" (misinterpreting the sound of letters when transforming a group of letters into a sound, e.g. "sympathy" read as "symphony"). Albert [9] concluded that such neurolinguistic studies suggest that the alexias can be subdivided along linguistic lines "... each reflecting a disruption of the reading process at a different linguistic level ... . caused by different... pathophysiologic mechanisms ...". This suggests that it is unlikely that the pathways (described below and demonstrated in Fig. 3) which appear disrupted in patients with alexia without agraphia are actually as simple as described. Recent work suggests that multiple regions of the brain probably interact simultaneously to perform most complex functions such as reading. These newer concepts of proposed interactions between different brain regions have recently been reviewed by Mesulam [11]. However, the exact anatomic location(s) of the lesions associated with the different subtypes of alexia have not yet been definitely identified. Therefore, the presumed location of a lesion that results in alexia without agraphia remains the inferior portion of the splenium of the corpus callosum.

\section{Anatomy (Fig.3)}

The dominant angular gyrus (language center) is located in the inferior parietal lobule of a cerebral hemisphere, usually the left, and receives direct input from the left visual cortex. Input from the right visual cortex reaches the left angular gyrus through white matter tracts that arise in the right occipital region, travel in the forceps major (white matter fiber tracts that interconnect the occipital lobes through the splenium of the corpus callosum), cross the midline in the splenium of the corpus callosum and project to the left angular gyrus through the deep white matter of the left parietal lobe. The left posterior cerebral artery supplies, among other structures, the left side of the splenium of the corpus callosum (via posterior pericallo- sal branches arising in the region of the quadrigeminal plate cistern) and the left visual cortex (via calcarine and parieto-occipital branches) [12].

\section{Pathology}

In reported cases of alexia without agraphia, infarction in the distribution of the left posterior cerebral artery (PCA) (Fig. 3a) it the most common pathologic process [13-15]. The left visual cortex is supplied directly by the left PCA and compromise of this artery therefore results in varying degrees of infarction of this region, destroying input from this area to the angular gyrus and causing the right homonymous hemianopia commonly associated with this syndrome. Infarction of a portion of the splenium of the corpus callosum affects the fiber tracts from the right visual cortex to the left angular gyrus without affecting the right visual cortex itself. Therefore, the end result is that vision (the right visual cortex) and language in the form of speech and writing (left angular gyrus and the motor cortex and its projections) are spared, but the patient is unable to read, as visual information cannot be transmitted to (has been "disconnected" from) the language area.

In the rare reported cases of alexia without agraphia not associated with a right homonymous hemianopia $[4$, 16], it is not hard to conceive of a fiber tract from the left visual cortex to the left angular gyrus being selectively affected with sparing of the left visual cortex itself while a second lesion selectively ablates the fibers from the right visual cortex. Alternatively, a single lesion proximal to the language area affecting the fiber tracts from both visual cortices (Fig. 3 b) might also cause alexia without agraphia without homonymous hemianopia.

Depending on the extent of the lesion, other neurologic abnormalities can be found, as in our two cases. These can include vision-specific anomia, impaired facial discrimination, difficulty with memory of presented visual material and disturbances of recent memory as well as motor, sensory and cerebellar abnormalities.

\section{Etiology}

While most cases of this syndrome have been reported in the setting of ischemic or thromboembolic disease [13-15, 17-19], cases associated with transtentorial herniation [20], migraine [21], intra- and extraaxial left occipital region neoplasms $[4,14-16,22]$ and surgery for a left occipital lobe vascular malformation [23] have also been reported, as have cases in the setting of carbon monoxide poisoning [24] and cerebral abscess [25]. Theoretically, any process (e. g. multiple metastases, traumatic shear hemorrhages, demyelinating plaques, etc.) affecting the splenium of the corpus callosum and related white matter tracts could result in this syndrome.

The two cases presented here are dramatic examples of the most frequent cause of alexia without agraphia, thromboemoblic disease involving the left PCA. However, interpreters of CT and MR examinations need to be aware of the underlying anatomic basis for this syndrome so that more subtle lesions may be sought in the appropri- 

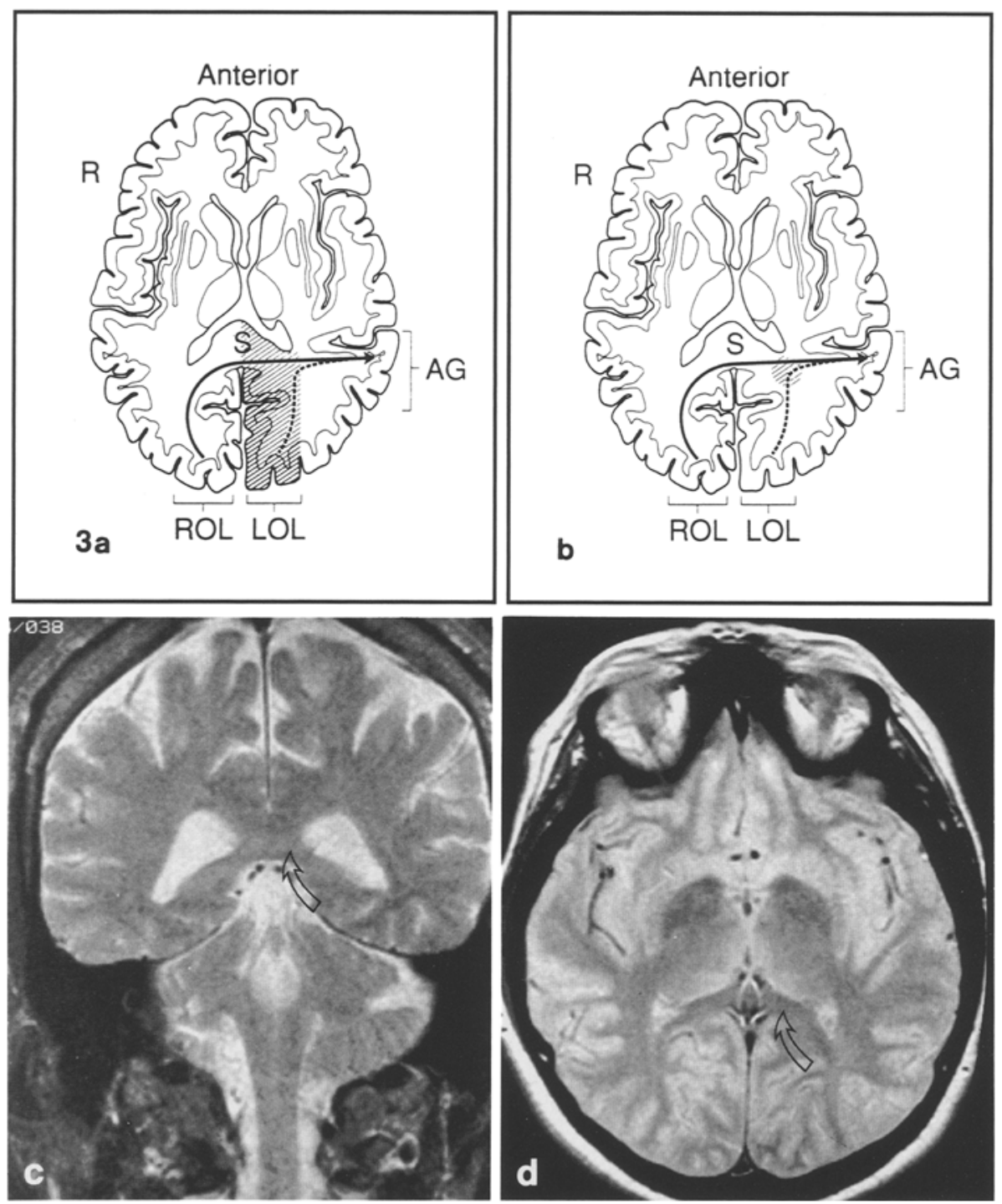

Fig.3a-d. Alexia without agraphia pertinent anatomy.

a Axial drawing demonstrating how a left posterior cerebral artery territory infarct (cross-hatched area) results in alexia without agraphia and a right homonymous hemianopia.

b Axial drawing of the site of a theoretical lesion (cross-hatched area) which could result in alexia without agraphia without a homonymous hemianopia.

c,d Normal coronal T2-weighted (3000/90) (c) and axial proton density weighted (3000/30) (d) $1.5 \mathrm{~T}$ MR images demonstrating the inferior portion of the left side of the splenium of the corpus callosum (arrow).

$A G$, Angular gyrus; $L O L$, left occipital lobe; $R$, right; $R O L$, right occipital lobe; $S$, splenium of corpus callosum; dashed line, fibers projecting directly from LOL to $\mathrm{AG}$; solid line, fibers projecting from ROL to AG through the inferior portion of the splenium of corpus callosum.

( $\mathbf{a}$ and $\mathbf{b}$ redrawn after [2]) ate clinical setting: images should be carefully reviewed with special attention to the region of the inferior portion of the splenium of the corpus callosum (Fig. 3c, 3d) and the deeper portion of the left occipital lobe.

Acknowledgement. We thank Karen Kluin, M.S., for her assistance with this manuscript.

\section{References}

1. Adams RD, Victor M (1989) Principles of neurology, 4th edn. McGraw-Hill, New York, pp 347-395

2. Nolte J (1981) The human brain, an introduction to its functional anatomy. Mosby, St. Louis, pp 279-281

3. Déjerine J (1892) Contribution a l'étude anato-pathologique et clinique des différentes variétés de cécité verbale. CR Soc Biol (Paris) 4: 61-90

4. Greenblatt SH (1973) Alexia without agraphia or hemianopsia. Brain 96: 307-316

5. Geschwind N (1965) Disconnection syndromes in animals and man. Brain 88: 237-294

6. Fincham RW, Nibbelink DW, Aschenbrenner CA (1975) Alexia with left homonymous hemianopia without agraphia. Neurology 25: 1164-1168
7. Benson DF (1979) Alexia. In: Benson DF (ed) Aphasia, alexia and agraphia. Churchill Livingstone, New York, pp 107-120

8. Mesulam M (1985) Principles of behavioral neurology. Davis, Philadelphia

9. Albert ML (1979) Alexia. In: Heilman KM, Valenstein E (eds) Clinical neuropsychology. Oxford University Press, New York, pp 59-91

10. Marshall JC, Newcombe F (1966) Syntactic and semantic errors in paralexia. Neuropsychologia 4: 169-176

11. Mesulam M (1990) Large-scale neurocognitive networks and distributed processing for attention, language, and memory. Ann Neurol 28: 597-613

12. Margolis MT, Newton TH, Hoyt WF (1974) The posterior cerebral artery, section 11. Gross and roentgenographic anatomy. In: Newton TH, Potts DG (eds) Radiology of the skull and brain, vol. 2. Mosby, St. Louis, pp 1551-1627

13. Johansson T, Fahlgren H (1979) Alexia without agraphia: lateral and medial infarction of left occipital lobe. Neurology 29:390 393

14. Levin HS, Rose JE (1979) Alexia without agraphia in a musician after transcallosal removal of a left intraventricular meningioma. Neurosurgery $4: 168-174$

15. Turgman J, Goldhammer Y, Braham J (1979) Alexia, without agraphia, due to brain tumor: a reversible syndrome. Ann Neurol $6: 265-268$ 
16. Vincent FM, Sadowsky CH, Saunders RL, Reeves AG (1977) Alexia without agraphia, hemianopia or color-naming defect: disconnection syndrome. Neurology 27: 689-691

17. Damasio AR, Damasio H (1983) The anatomic basis of pure alexia. Neurology 33: 1573-1583

18. Naranjo IC, Espada JL, Torrella JE, Donderis MAC, De las Penas R (1989) Alexia without agraphia: a new case studied by CT-scan. Neuroradiology 31: 199

19. Weisberg LA, Wall M (1987) Alexia without agraphia: clinicalcomputed tomographic correlations. Neuroradiology 29: 283 286

20. Kirshner HS, Staller J, Webb W, Sachs P (1982) Transtentorial herniation with posterior cerebral artery territory infarction. A new mechanism of the syndrome of alexia without agraphia. Stroke 13: 243-246

21. Bigley GK, Sharp FR (1983) Reversible alexia without agraphia due to migraine. Arch Neurol 40:114-115
22. Cohen DN, Salanga VD, Hully W, Steinberg MC, Hardy RW (1976) Alexia without agraphia. Neurology 26:455-459

23. Ajax ET, Schenkenberg T, Kosteljanetz M (1977) Alexia without agraphia and the inferior splenium. Neurology 27:685-688

24. Kawamura M, Ito N, Hirayama K (1981) Alexia without agraphia or hemianopsia in a case of CO intoxication (author's trans. lation). Rinsho Shinkeigaku 21: 628-636

25. Leiguarda R, Carrea R (1977) Alexia sin agrafia (correlación clinicotomográphica). Acta Neurol Latinoam 23: 89-101

Dr. D.J.Quint

Divison of Neuroradiology

Department of Radiology

University of Michigan Hospitals

1500 East Medical Center Drive

Ann Arbor, Michigan, 48109-0030

USA 